\title{
Kirche und Presse in den Entwicklungsländern ${ }^{1}$
}

von Joseph-Roger de Benoist

Mit dem, was hier kurz dargelegt wird, sollen keine theoretisch-abstrakten Ansichten über die Rolle der Kirche bei der Förderung und Bewertung der „Massenmedien“ in den Entwicklungsländern ausgebreitet werden, sondern es werden nur einige Punkte aus der Sicht der praktischen Erfahrung erörtert.

\section{Die vorbandenen Publikationen erbalten}

Man muß sich Mühe geben, die vorhandenen Publikationen weiter zu entwickeln, vor allem jene, die international akzeptiert sind. Das ständige Aufdrängen von Subventionen ist jedoch nicht das beste Mittel, um diese Publikationen zu unterstützen. Ohne Hoffnung auf einen möglichen Haushaltsausgleich in allen Fällen muß man eine Politik der Gleichgültigkeit (gegenüber den bevormundenden Behörden) ebenso wie eine Politik der Mühelosigkeit (gegenüber dem Zeitungspersonal) vermeiden. Die Betriebs- und Nutzungsbedingungen lassen sich verbessern:

- Wenn die Presseorgane und Nachrichtenagenturen der entwickelten Länder den Zeitungen in den Entwicklungsländern in beruflicher Solidarität helfen, ihre publizitären Einnahmen zu vermehren;

- wenn die finanzielle Verwaltung streng geführt wird;

- wenn die Leitung die Sorge hat, die Zeitung den sozialen, wirtschaftlichen, politischen und kulturellen Bedingungen, die in ständiger Entwicklung sind, unablässig anzupassen. Das ist leider nicht immer der Fall: Es gibt Periodika, die ihr Erscheinungsmuster seit zehn Jahren kaum geändert haben, während die eben genannten Bedingungen sich beträchtlich gewandelt haben.

Daß das Eigentum oder das Patronat dieser Zeitungen oft in den Händen der Hierarchie liegt, die sich als wenig kompetent erweist und folglich kaum Ansprüche in Pressefragen erheben kann, begünstigt bisweilen eine gewisse Lässigkeit auf Seiten des Redaktionsteams. Es wäre zu wünschen, daß das Eigentum oder zumindest die Úberwachung der Presseorgane kompetenten Laien zufiele, die die Redaktion zu überprüfen und anzuregen imstande sind.

Auf jeden Fall ist es der Mühe wert, finanzielle und personelle Opfer einzubringen, um jene Periodika zu erhalten, die bereits eine bedeutende Rolle gespielt haben und deren Name in weiten Gebieten bestens bekannt ist.

\section{Nationale Zeitungen fördern}

Es erscheint wünschenswert, daß es in jedem Land eine katholische allgemein informierende Publikation ganz gleich welcher Art gibt. Die Christen brauchen ein Forum, von dem aus sie über die wesentlichen Tatsachen und Probleme in ihrem Land ihr

Joseph-Roger de Benoist, W. V., war langjähriger Redakteur und Direktor der katholischen Wochenschrift Westafrikas "Afrique Nouvelle“ in Dakar, Redakteur der Zeitschrift „Vivante Afrique" in Namur und gründete 1967 die gemeinsame französische Missionszeitschrift "Peuples du Monde"; heute leitet er die Diözesan-Caritaswerke in Cotonou in Dahomey. 
Urteil abgeben können. Alle gutwilligen Menschen haben eine Zeitung nötig, der sie ihr Vertrauen schenken, da sie wissen, daß sie sich um Objektivität müht und von der obrigkeitlichen Macht nicht abhängt. Die Zeitungen mit internationaler Verbreitung können diese Rolle nicht übernehmen: Die Informationsverzögerung, die Verzögerungen von Herstellung und Versand hindern sie daran, dem aktuellen Geschehen wirklich zu folgen. Auch haben sie nicht immer an Ort und Stelle einen Korrespondenten, der objektive Informationen zu übermitteln imstande ist.

\section{Die Presse der Apostolatsbewegungen entwickeln}

Die Erfahrung zeigt, daß die Verbandsblätter eine sehr große Rolle für die Lebenskraft und Entfaltung der verschiedenen Laienapostolatsbewegungen spielen können. Diese Blätter können bescheiden sein und sich auf einige vervielfältigte Seiten beschränken, falls sie zwei Voraussetzungen erfüllen :

- Wenn sie einen entsprechenden Inhalt haben, der das konkrete Leben und die Probleme des Landes widerspiegelt;

- wenn sie mit gleichbleibender Regelmäßigkeit erscheinen.

\section{Beziebungen zur profanen Presse unterbalten}

Es ist sehr nützlich, daß ein dazu befähigter Priester oder Laie beauftragt wird, Beziehungen zu Presse und Rundfunk wahrzunehmen:

- Damit man zur gegebenen Zeit den Agenturen, Zeitungen und Rundfunksendern die das Leben der Kirche betreffenden Informationen übermitteln kann;

- damit man die Arbeit der Reporter bei religiösen Ereignissen erleichtert;

- damit man gegebenenfalls durch Artikel oder Sendungen mit dazu beiträgt.

Die Erfahrung lehrt, daß die Berufsjournalisten gern einen vermittelnden Sprecher haben, der über ihre Probleme auf dem laufenden ist. Freundschaftliche Beziehungen zu ihnen ermöglichen oft die Benutzung dieser Informationsmittel und helfen das Denken der Kirche viel breiter zu streuen, als die katholische Presse es kann.

Wenn dieser "Pressebeauftragte" regelmäßig mit Zeitungen und Agenturen im Ausland zusammenarbeiten soll, ist es normal, daß seine Dienste bezahlt werden. Das ist nicht mehr als recht und das einzige Mittel, eine regelmäßige Mitarbeit sicherzustellen.

\section{Handzettel und Traktate veröffentlichen}

Wenn es nicht immer möglich ist, das regelmäßige Erscheinen einer katholischen Zeitung aufrechtzuerhalten, ist es wünschenswert, mit Traktaten, Broschüren, Handzetteln ohne feste Periodizität auf die Fragen zu antworten, die sich Christen und Nichtchristen stellen. Da die Verbreitung dieser Traktate großenteils vom Verkaufspreis abhängt, scheint es angemessen, den Herstellungspreis durch eine unberechtigt hohe Auflage zu vermindern, die man jedoch oft nur durch die Zusammenarbeit mehrerer Länder ins Auge fassen kann.

Die Anstrengungen in dieser Richtung, die der „Verlag St. Paul“ in Abidjan (Elfenbeinküste) mit der Publikation „Pirogue“ unternahm, ist lobenswert. Diese Vierteljahresschrift, deren einzelne Ausgabe jeweils einem einzigen Problem gewidmet ist, 
kann in allen Ländern des französischsprachigen Afrika verbreitet werden. Der Erfolg der ersten Ausgabe beweist, daß sie einem notwendigen Bedarf entgegenkommt. Sie wird sich halten, wenn die Redaktion, wie die Initiatoren es vorsehen, von Personen wahrgenommen wird, die in Afrika wohnen und deren Text Revisoren mehrerer afrikanischer Länder vorgelegt wird. So ist gewährleistet, daß man ein großes Publikum erreicht.

\section{Druckereien modernisieren oder schaffen}

Eine Druckerei ist immer eine nützliche Stütze für das Apostolat durch die sozialen Kommunikationsmittel. Aber man muß zwei Fälle unterscheiden:

Dort, wo eine alte, oft bedeutende Druckerei vorhanden ist, die auch kaufmännischgeschäftliche Arbeiten ausführen kann, müßte man bestrebt sein, sie eher zu modernisieren als auszubauen. Viele Druckereien sind, weil sie versäumt haben, ihren $\mathrm{Ma}$ schinenpark zu erneuern, von neueren Geschäftsdruckereien und oft auch durch staatliche Druckereien überholt worden. Diese sind fast in allen Ländern vorhanden, und sie führen alle Verwaltungsdrucke aus, die ehemals den Missionsdrudkereien zufielen. Besser ist es in solch einem Fall, sich mit den nationalen Druckereien ins Benehmen zu setzen, um ihnen gegebenenfalls die bedeutendsten und ältesten Maschinen abzutreten, die oft unbenutzt oder unausgelastet sind, und die katholische Druckerei auf bescheidenere Ausmaße zu beschränken, aber mit modernen, dem wirklichen Bedarf angepaßten Mitteln auszustatten.

Wo es keine Druckerei gibt, wäre es gut, eine zu begründen, die in ihren Zielen begrenzt ist, aber ausgerüstet mit modernem Material alles Wesentliche an katholischer Publikation leisten kann: Zeitungen, Blätter der Apostolatsbewegungen, Traktate, Katechismus, liturgische Bücher in den Ortssprachen usw. Es scheint, daß die Offsetmaschinen am ehesten der Gesamtheit dieser Aufgaben entsprechen.

Die Hierarchie dürfte nicht allein interessiert sein an der Gründung und Verwaltung dieser Druckereien. Die Gesellschaften könnten mit kompetenten Laien besetzt werden.2

\section{Kompetente Journalisten ausbilden}

Muß die Kirche sich selbst um die Ausbildung von Berufsjournalisten bemühen? Das ist nicht so sicher. $\mathrm{Zu}$ oft kehren diese Journalisten bei Rückkehr von den Schulen, wohin sie geschickt wurden, nicht mehr in den Dienst der katholischen Presse zurück. Gewiß ist die Bemïhung nicht zwecklos: Es ist gut, daß es in der profanen Presse katholische kompetente Journalisten gibt. Wenn man sich indes gegen dieses Mißgeschick wappnen will, muß man eindeutige und juristisch gültige Verträge abschließen.

Es scheint jedoch, daß die katholische Presse das kompetente Personal unter zwei Bedingungen leicht fände:

- Wenn die Journalisten, die sie beschäftigt, korrekt bezahlt würden;

- wenn ihr Berufsstand genau umschrieben würde: Wenn die Hierarchie sich auch normalerweise damit befassen muß, daß die Presse, die sich katholisch nennt, im wesentlichen die Positionen der Kirche widerspiegelt, so muß der katholische Journalist doch eine gewisse Bewegungsfreiheit haben und darf nicht nur ein schlechthin ausführendes Organ sein. 
Dieser letzte Punkt ist vielleicht jener, der das größte Problem aufgibt. Die katholische Zeitung kann ihre Rolle nur spielen, wenn sie nicht das ausschließliche Forum des Bischofs und der getreue Spiegel aller kirchlichen Feiern ist und dabei jedes wirtschaftliche und politische Problem zurückdrängt. Ein Diözesanbulletin muß diese Rolle übernehmen und die katholische Zeitung davon entlasten. Diese kann dann "engagiert" sein und wirklich und umfassend das Leben nicht nur der Christen, sondern aller Bewohner des Landes spiegeln. Das setzt voraus, daß die Laienredakteure sich frei und in eigener Verantwortung äußer können: Das ist die unabdingbare Voraussetzung, damit die Publikation ein gewisses Publikum außerhalb der mehr oder minder begrenzten christlichen Gemeinschaft findet. Ist die katholische Zeitung nicht das Mittel, das Wort Gottes im "Alltagsgewand“ allen Menschen guten Willens darzulegen?

\section{Anmerkungen:}

1. Joseph-Roger de Benoist schrieb diesen Text als Vorbereitungspapier für den Trierer Vorkongreß der Entwicklungsländer vom 12. bis 14. Juli 1971 zum Weltkongreß der UCIP in Luxemburg; vgl. CS 4: 1971, 341-343.

2. Was die Druckereifrage angeht, vgl. den Bericht des Verfassers vom Oktober 1963 über die katholischen Druckereien in den französischsprachigen Ländern Afrikas, herausgegeben von der UCIP auf Antrag des französischen Ministeriums für Zusammenarbeit.

\section{SUMMAR Y}

The author offers short recommendations out of practice for the religious Press in developing countries. The existent publications should be kept if possible and be made independent by competent journalists. All developing countries should have a generally informing $\mathrm{Ca}-$ tholic newspaper. The smaller perriodicals of the apostolate too play an important role according to the author. He also gives great importance to the relations with the secular Press. Where no Catholic paper can be edited regularly, there pamphlets are to be favoured. The autor is recommending modern, capable printing-offices. Formation of journalists under clear contracts - shall create lay editors, being allowed to speak frankly and in own responsibility.

\section{R E S U MEN}

El autor da sucintas recomendaciones de la práctica para la prensa religiosa en los países en vías de desarrollo. Las publicaciones existentes deben en lo posible quedar y estar hechas independentes por periodistas competentes. Todos los países en vías de desarrollo deben tener un periódico católico informando generalmente. También a los periódicos del apostolado el autor atribuye un papel importante. A las relaciones con la prensa secular da gran importancia. Se debe favorecer folletos en regiones, donde no puede publicarse un periódico católico. El autor recomenda imprentas capaces y modernas. Una formación periodística por contratos claros - debe proveer redactores laicos, quienes pueden expresarse francamente y en propia responsabilidad. 